\title{
Self-organizing technique for improving coverage in connected mobile objects networks
}

\author{
Sofiane Hamrioui $^{1} \cdot$ Pascal Lorenz $^{2}$ (I) $\cdot$ Jaime Lloret $^{3}$
}

Published online: 9 May 2017

(C) Springer Science+Business Media New York 2017

\begin{abstract}
Despite the multiple benefits offered today by connected mobile objects networks (CMONs), some constraints continue to limit their development and to degrade their applications and services' performance. Given their limited energy, some or many objects may stop functioning which leads to the deterioration of network functionalities such as monitoring, detection and transfer of data. It is in this context that our work is situated, namely the improvement of applications performance and the quality of service (QoS) within CMONs, by exploiting some communication environment parameters and geometry techniques. We propose a new technique called self-organization area coverage (SOAC) for CMONs which aims to ensure maximum coverage in the network while optimizing the exploited resources. SOAC has been evaluated and compared not only to the network without improvement but to two other solutions proposed in the literature. The obtained results show a clear improvement in terms of network coverage and several QoS parameters.
\end{abstract}

Keywords Connected mobile objects networks - Selforganizing $\cdot$ Network coverage $\cdot$ Mobility $\cdot$ Communication range $\cdot$ QoS $\cdot$ Energy effectiveness

\footnotetext{
B Pascal Lorenz

lorenz@ieee.org

Sofiane Hamrioui

sofiane.hamrioui@uha.fr

Jaime Lloret

jlloret@dcom.upv.es

1 GRTC/MIPS, LMIA, University of Haute Alsace, Mulhouse, France

2 GRTC/MIPS, University of Haute Alsace, Mulhouse, France

3 Universidad Politecnica de Valencia, Valencia, Spain
}

\section{Introduction}

Several intelligent networks and systems have emerged recently (like smart water networks, smart grid, smart cities and homes, intelligent transportation) which they are connecting all our world more than we ever thought possible. The common characteristic of such systems and networks is the exploitation of wireless communications to ensure the connections between the different objects able to be mobile [1-3]. The emergence of CMONs is one of the most important advances in technology that the world of networks and telecommunications has recorded in these last years. Such environment could be conceptually defined as a dynamic network with self-configuring capabilities. It is based on standard and interoperable communication protocols. In these networks, every physical or virtual object has its own identity and attributes. Intelligent interfaces are used by all these objects which are seamlessly integrated into the information network [4]. The meaning of objects here implies their ability to be intelligent and the diversity of their nature (devices, sensors, robots, drones, etc) [5]. For the rest of our paper, the different objects of this network will be designated by one more generic word which is node.

Despite all the advantages offered by CMONs, they are faced with new challenges that slow down their evolution and degrade the performance of their applications. With the limited energy and the mobility of the objects, changes in the physical topology of the network occur frequently, leading in most cases to the appearance of uncovered areas that become isolated from the rest of the network. This justifies the need to design new protocols and techniques enabling the selforganization of the network in order to ensure better coverage and avoid the services interruption for CMONs applications. It is the objective of the work carried out in this paper through which we propose a new technique to maintain good network 
coverage by exploiting the communication parameters of the objects and geometric calculations. The rest of the paper is organized into six sections. We discuss the problem definition in Sect. 2. Section 3 presents some research works conducted in literature and addressing the same issue as our work. Our proposed technique is given in Sects. 4 and 5 are devoted to its evaluation. The conclusion of the paper is given in Sect. 6 with some perspectives on our future work.

\section{Problem definition}

CMONs nowadays offer new perspectives in the area of telecommunications and do not pose any restrictions on the location of users due to the use of radio waves instead of wired infrastructure. This new mode of communication creates new specific features to the wireless environments mainly frequent disconnections, modest resources and communication rate, limited energy, degraded quality services, inefficient security, etc. In CMONs, the different objects are designed to operate for months or years (for example in the context of e-health). This is why the energy capacity of these objects must be used efficiently to maximize network lifetime. In fact, an object without enough energy is considered defective and therefore can not meet the requirements of the network services. If this problem is generalized to many objects, a change in the network topology appears creating then uncovered areas and connectivity breaks between the objects which are still working.

To overcome this problem, the nodes belonging to the network must be able to self-organize themselves in order to maintain maximum network coverage. This task can be done by acting on the communication parameters and ensuring optimization of energy consumption in the network. A self-organization of the network allows the continuity of its activities (such as monitoring, assistance, computing, etc). Only some objects from logical topology should be involved in the self-organization in order to cover the empty area appearing in the network. The form of this area is often known (such as square or a circle for example) but it can be also a complex form. In the case where the form of the uncovered area is complex (not known), geometric methods exist which can divide this form and transform it into two more known forms, which leads us to the first case. To accomplish the task of self-organization, and as part of our work, we are interested in two communication parameters namely coverage range (communication range) of the objects and their mobility.

These last few years we have been witnessing the emergence of many miniaturized and mobile devices that can cooperate in ad hoc manner without any previous control infrastructure. These factors allow us then to consider mobility as a fundamental aspect of self-organization in CMONs. Mainly, three mobility classes are considered: ran- dom mobility, predictable mobility and controlled mobility [6-8]. Controlled mobility allows the nodes to move from a source to a destination to accomplish specific objectives. This mobility varies according to a defined model in advance. This type of mobility has several advantages essentially good coverage management, the reduction of consumed energy and the improvement of the communication protocols performance. For these reasons, we consider this type of mobility as one way to exploit in the context of self-organization and maintain it between the connected objects and for a better performance of CMONs applications.

In environments such as CMONs, to solve the problem of connectivity break and cover the empty areas which appear we can proceed in three different ways. The first way is to use mobility (as explained in the previous section). The second way is to extend the network by adding new objects as to ensure maximum coverage and then fill the gaps. And the third way is to adapt the coverage radius (communication range) of some objects in order to cover the uncovered area. However, the choice of some objects among the others in order to adjust their coverage rays should be done by keeping equitable energy levels for all of them. In fact, our work involves the exploitation of the two communication parameters just presented and geometric methods to allow an intelligent control of network topology and its selforganization to ensure maximum coverage. Our proposed SOAC aims to ensure maximum coverage in the network while optimizing the exploited resources. We act directly on the connected objects by changing two of their parameters which are mobility and coverage range.

\section{Related work}

The majority of the conducted works in the literature in the context of self-organization and network coverage have not yet addressed the case of CMONs. Some of them are carried out around solutions for better performance of WSNs [9-14]. However, both type of networks, CMONs and WSNs, have common characteristics and they are confronted to almost the same constrains. In both cases the problem of network coverage occurs and the proposed solutions should take into account the fact that the energy of the objects (which can be sensors) is very limited. These works addressed the specific problems of such systems essentially the limited energy of the connected objects and the network coverage. Indeed, the objects can be deployed in an "ad hoc" way, for this reason the network has to deal with the uncovered areas and also should be self-organized. In addition to that, as each object has a limited energy, the whole network should minimize the total energy consumed to allow autonomous operation without any assistance in order to prolong and extend the network lifetime. We present in this section some existing 
techniques and schemes addressing the same problem as the one dealt within this document.

\subsection{Spanning hyper-tree algorithm (SHTa)}

Based on the hyper-graph theory, SHTa is proposed in [15] in order to compute the minimum transmitting power delivery paths set for WSNs coverage cast. The authors present a novel hyper-graph model to abstract large-scale and high connectivity WSNs (wireless sensors networks) into a robust hyper-tree infrastructure. They present also a precise mathematical derivation that solves the "hyper-tree existence" problem. SHTa has been proposed to compute the delivery paths set, which is the minimum power transmitting coverage cast hyper-tree. Variable scale hyper-edges represented as computing units limit solution space and reduce computing complexity. Mutual backup delivery paths in one hyper-edge improve the capability of fault tolerance. In fact, fault avoiding allows maintaining the largest number of nodes in the network which leads then to ensure optimal network coverage. The weak point in the proposed solution is the fact that it considers only the power parameter in the determination of the best path. Also, only the case of static sensors devices is considered.

\subsection{Backup nodes self-organization scheme}

As in the case of SHTa, in order to ensure good network coverage by keeping functional (without fault) the nodes within the network, a backup nodes self-organization scheme is described in [16]. This scheme designates a set of backup nodes to replace failed nodes and maintain a backbone for coverage and communication. The proposed scheme does not require a centralized server for monitoring of node failures and for the designation of backup nodes to replace failed nodes. It operates in a fully distributed manner and it requires only localized communication. This scheme has been implemented on top of an energy-efficient selforganization technique for sensor networks. The proposed fault-tolerance-node selection procedure can tolerate a large number of node failures using only localized communication, without losing either sensing coverage or communication connectivity. As in the previous solution, the authors treat only the case of sensors networks without considering possible mobility of the sensors. Also, the fault tolerance aspect considered by the proposed solution did not take into account the different sources of these possible faults.

\subsection{Self-organized, flexible, latency and energy efficient (SOFLEE) protocol}

To ensure an efficient consumption of energy and to avoid bad network coverage caused by the depletion of energy of some nodes, SOFLEE protocol is proposed in [17]. This protocol is designed based on cross-layer approach for wireless sensor networks that uses time division multiple access (TDMA) based medium access scheme combined with multihop routing information during time slot allocation. Time slot allocation is done centrally by master station (MS) to provide a collision-free and fair media access. MS allocates same transmission slot to nodes that are two hops apart to increase channel spatial reuse and decrease data latency. For data gathering at MS, SOFLEE uses parenthood willingness to forward data to MS through unidirectional tree rooted at MS. The parenthood willingness requires simple comparisons against thresholds, and thus, it is very simple to implement on memory and computationally constrained nodes. One important weak aspect of the proposed solution here is the use of TDMA as medium access scheme while most part of the wireless networks uses other technologies.

\subsection{Multi agent system based on organizational theory}

A poor management of the exchanged information between the nodes within heterogeneous distributed sensor networks and their processing in an inefficient way can be the cause of no optimal coverage. In order to overcome this problem, a solution to automatically fusing the information coming from different sources in an efficient and effective manner is proposed in [18]. The authors present a multiagent system that based on the organizational theory and propose a new model to automatically process and fuse the information in heterogeneous distributed sensor networks. The proposed architecture is applied to a case study for indoor location where information is taken from different heterogeneous sensors. Using information management to reduce the energy consumption does not guarantee the change of topology in the network. Adding to this, the proposed solution deals with the case of sensor networks and assuming that the sensors are not mobile.

\subsection{Reliability, energy and delay with ant colony optimization (RED-ACO) technique}

Optimization systems exploiting ant colony have been used in several research works due to their ability to always produce good results in terms of organization and network coverage. It's in this context that a new technique, called RED-ACO technique has been proposed in [19]. RED-ACO aims to combine QoS based routing metric with ant colony optimization (ACO). The objective of this technique is to ensure efficient routing in terms of quality of service while maintaining optimal network coverage and always having a possible path to all the nodes belonging to the network. The problem of providing QoS routing is formulated as link-based and path-based metrics. The search range for an ant to select its next-hop 
sensor is limited to a subset of the set of the neighbors of the current sensor based on the required metric values. The proposed solution recorded good results for the homogeneous environment but becomes inefficient even nonfunctional for heterogeneous environment with mobility of objects. In addition to that, only network layer is considered by the authors by ignoring important communication aspects such as the distance between sensors.

\subsection{Heterogeneous clustering and energy consumption algorithm}

To maximize network coverage, prolonging the nodes lifetime is one way which can be exploited. This issue is explored by taking into account the nature of heterogeneous wireless sensor networks. The result of the investigated studies is the proposition of a novel algorithm to address the problem of finding an effective pathway for heterogeneous clustering energy [20]. The proposed algorithm implements cluster head selection according to the degree of energy attenuation during the network's running and the degree of candidate nodes' effective coverage on the whole network, so as to obtain an even energy consumption over the whole network for the situation with high degree of coverage. The authors treat only the case of sensors networks without considering possible mobility of the sensors. The lifetime aspect considered by the proposed solution did not take into account the different sources that can influence this parameter (like distance, mobility, data losses, etc). Also, the authors assume that there are enough sensors in the network which is not true in real situations.

\subsection{Self-organized node movement technique}

A simple and novel technique for self-organized node movement to satisfy the coverage of the given region of interest is proposed in [10]. This solution supposes an initial random deployment of mobile sensor nodes and uses a least number of nodes, such that the maximum node displacement is minimized. The authors present a simple centralized algorithm and also a distributed version of it for node placement. Moreover, in case of a node failure, a distributed fault recovery algorithm is proposed to replace it locally utilizing the available free nodes. The solution proposed here also deals with the case of static and no-mobility sensors. Adding to this, the authors concentrate on the stage of deployment of the sensors and assume that there are enough sensors to be able to replace those that do not cover their zones. Another weak aspect is the fact that the authors have not proposed an efficient process for the selection of the sensors among those that are available and did not take into account their specific parameters in this process.

\subsection{Movement-assisted technique for nodes self-deployment}

To control the mobility of nodes and make them cover as much as possible the geographical area of the network, a neural network model is used in [21]. In addition to that, the authors consider also a genetic algorithm for the training of the neural network through reinforcement learning. The authors propose a movement-assisted technique for nodes self-deployment by focusing on the self-organizing capabilities of nodes in WSRN. This kind of scheme is extremely adaptive, since it can be easily modified in order to consider different objective and/or QoS parameters. In fact, it is sufficient to consider a different input for the neural network to aim to a different objective. The authors proposed a new method for programming a WSRN and showed practically how the technique works, when the coverage of the network is the QoS parameter to optimize. The proposed solution allows a self-deployment of the different robots and then initially the coverage is ensured but such algorithm does not allow self-organizing according to the new parameters of the robots. Another weak aspect is the fact that the adaptation to the evolution of the network is not considered by the proposed solution which, in presence of a massive quantity of obstacles, becomes inefficient.

\subsection{Particle swarm optimization (PSO) algorithm}

One of the difficulties to overcome in sensor networks is the efficient deployment of the sensors and their positioning in a way to ensure total coverage of the network. This problem is well investigated in [22] through which the authors proved that an optimum deployment of the sensor nodes is essential for better utilization of the sensor network. They proposed a novel algorithm based on particle swarm optimization (PSO) in order to get good network coverage by keeping enough active sensors and by extending their lifetime. With an important lifetime for sensors and good coverage, the fault cases and the complexity of the network are reduced and also the QoS of applications is increased. The algorithm proposed here simplifies the network considerably by disregarding all the parameters making the communication environment more complex. The second weak aspect of this algorithm is the fact that it is devoid of any self-organizing mechanism.

Most of the proposed solutions treat the case of WSNs and they are not oriented to the CMONs that have begun to introduce recently a new characteristics not necessarily existing within WSNs. The source of energy that can be different for the nodes and their ability to process data are the two important features that distinguish the CMONs from the WSNs. Also, most of the proposed solutions are based on the use of existing protocols and algorithms by providing some improvements at a specific level of OSI model. Each 
one of the proposed solutions has its way to overcome the problem of network coverage. Some parameters from the communication environment have been taken into account by these solutions but they are exploited without regard to their optimum and efficient use. Our work is within the same context as those presented in this section and aims to propose an intelligent self-organizing system for the CMONs in order to ensure maximum coverage. The proposed solution takes into account the specific characteristics of CMONs in addition to those of WSNs. To consume the least amount of energy, the proposed solution is simple, distributed and does not require complex calculations. In addition to that, the proposed solution manages optimally the different parameters used to ensure maximum network coverage. Another strong aspect of our solution is the use of several layers of the OSI models, contrary to what was presented previously.

\section{Model of the proposed technique}

The goal of our work in this paper is to propose a technique for better network coverage and to improve the QoS for CMONs applications mainly in terms of energy consumption, network lifetime and end to end delay. In fact, such results can be reached especially in two ways. The first way is with avoiding the connectivity break in the network due mainly to the energy depletion of one or several nodes. The second way is by reducing data losses caused by the different types of interferences (collisions, channel errors, congestion, etc). As part of our work, we are interested to the first case namely ensuring a maximum coverage for the network by exploiting the self-organization of its nodes.

The proposed SOAC technique is implemented on physical and network layers by adding some metrics and treatments. The goal is to improve these two layers by making them more suitable for CMONs applications. At network layer level, we propose to allow the network to control the mobility of the nodes and select the most appropriate ones to move from their initial position to the specified destination. This task is accomplished according to some conditions to verify mainly the position and the energy level of the nodes. The second parameter on which SOAC is acting is the coverage radius (communication range) of some nodes in order to cover uncovered area. This treatment is ensured at physical level by keeping equitable energy levels for all nodes and maintaining, as much as possible, a high level of network coverage.

The approach used here is based on the exploitation of geometric and optimization calculations to allow an intelligent control of the network topology and its self-organization ensuring then a maximum coverage. Figure 1 gives the different steps of how the proposed solution works. For each event

$E v$ corresponding to the appearance of an uncovered area $U A$ in the network, a first treatment is performed by a procedure called DETECT_FORM() in order to determinate the geometric form which corresponds the most to this $U A$. In the case where the $U A$ does not correspond to any known geometric form, another procedure called SPLIT_FORM $(\mathrm{Nb})$ is performed whose role is to split this $U A$ and make appear $\mathrm{Nb}$ sub areas close to known geometric forms.

Once the UA form is known, and as shown in Fig. 1, a procedure called $\boldsymbol{R A D I U S}(\boldsymbol{R})$ is executed through two main tasks. The first one is the calculation of the center $C$ of the geometric form returned by the previous DETECT_FORM() procedure. The second task calculates the radius $R$, which is the distance between the center $C$ and the farthest point situated at the border of $U A$. The radius $R$ is compared to the communication range $P$ of the nodes. In the case where $R$ is inferior or equal to $P$, the $\boldsymbol{A D J U S T}(\boldsymbol{N d})$ procedure is executed otherwise another procedure called $\operatorname{MOVE}(\mathbf{N d}, \mathrm{Dst})$ is performed.

SOAC distinguishes two classes of data $C D$ manipulated by all the nodes. These data are defined as follows: $I$ for Important data and NI for no important data. In our context, important data means the data which need a good level of energy, to be treated and forwarded in real time and they are important for the good performance of the applications. In fact, two energy thresholds are defined, called $S_{I}$ and $S_{N I}$, to which the energy level $R_{E}$ of node $N d$ is compared. The procedure exploiting these two thresholds is $\boldsymbol{S E L E C T}(\boldsymbol{N} d$, $\boldsymbol{C S}$ ) and it basically uses the Eq. (1).

$$
C S:=\begin{array}{ll}
r_{R_{E}} \geq S_{I} & \text { if } C D_{N d}=I \\
R_{E} \geq S_{N I} & \text { if } C D_{N d}=N I
\end{array}
$$

where $C S$ is the selection condition and $C D_{N d}$ is the data class treated by the node $N d$.

The nodes which are working on data belonging to the class $I$ are not authorized to be selected by the procedure SELECT $(\boldsymbol{N d}, C S)$ if their level of energy $R_{E}$ is inferior to the $S_{I}$.

The ADJUST $(\boldsymbol{N d})$ procedure is responsible for adjustment of the communication range $P$ of the selected node $N d$. In the case where $(R<P)$ this procedure is applied to the nodes situated at the border of the $U A$ and this after the verification of their energy levels and the type of data processed $C D$. Indeed, the energy level $R_{E}$ of the nodes selected for the adjustment is compared to a given threshold, called $S_{A d}$. This comparison would determine how much to adjust the radius of each node. The main equation used by the procedure $\operatorname{ADJUSTS}(\boldsymbol{N d})$ is given here:

$$
P:=\begin{array}{ll}
\mathrm{r}_{P}+\frac{R-P}{L} & \text { if } R_{E} \geq S_{A d} \\
P+\frac{R-P}{2 L} & \text { if } R_{E} \leq S_{A d}
\end{array}
$$


Fig. 1 The functioning of SOAC technique

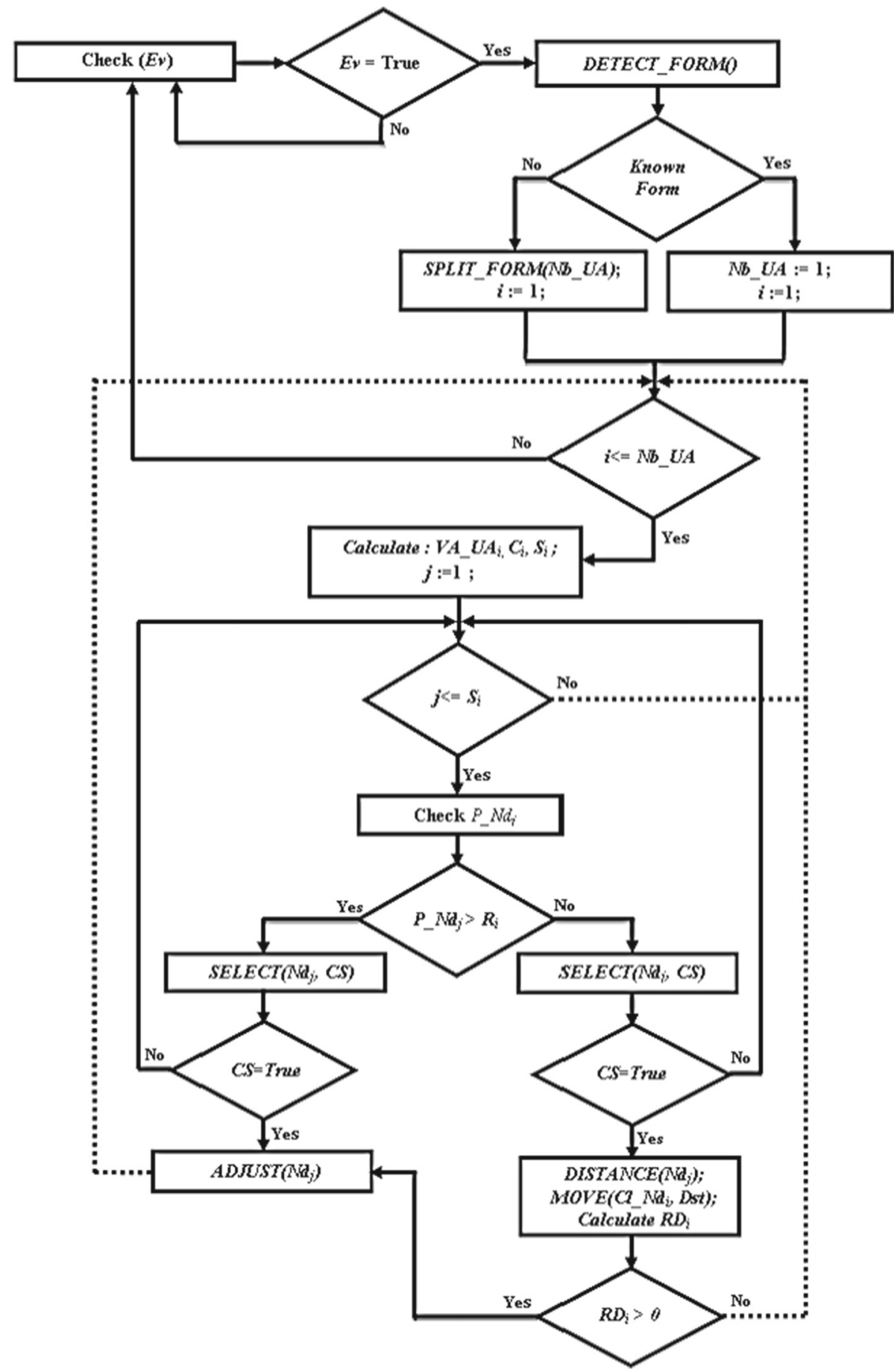

As we can see in Fig. 1, the procedure $\operatorname{MOVE}(\boldsymbol{N d}, D s t)$ is called in the case where the radius $R$ calculated above exceeds the range $P$. It is responsible for moving a node $N d$ from its initial position $P_{0}$ to a destination $D s t$ which is the center $C$ of the uncovered area $U A$.

Let $R D$ and $R S L$ two variables with:

$R=P^{*} R S L+R D$
The communication range $P$ of the node to be moved will be adjusted by a value equal to the entire part of $R D$.

The node to be moved is selected according to some conditions linked to its energy $R_{E}$, its distance from the center $C$ of the $U A$ and the $C D$ processed. In addition to the procedure $\operatorname{SELECT}(N d, C S)$ which is checking the two values $R_{E}$ and $C D$, a procedure $\boldsymbol{D I S T A N C E}(\boldsymbol{N d})$ is performed to find the closest nodes to the $U A$. 
The Algorithm 1 gives the main module of the SOAC solution that uses the different procedures previously discussed. The algorithm is started by the detection of an event $E v$ corresponding to the appearance of an uncovered area $U A$ in the network. In the case where the form of the $U A$ is not a known geometric form, it will be divided into two or more sub areas whose forms are known. Once all the uncovered areas have all known forms, each one will undergo the same treatment. This treatment starts with the calculation of the ray $R$ of the uncovered area to which the communication range
$P$ of every node from the $U A$ is compared. In the case where $P$ is smaller than $R$, the class of the data processed $C D$ by the node and its level of energy $R_{E}$ are checked too in order to know if $P$ can be adjusted or not. In the case where $P$ of the node is longer than $R$ and if this node is the closest to the center $C$ of the $U A$, it will be selected to be moved from its initial position to the center $C$. The communication range $P$ of the moved node can be also adjusted in the case where the rest of division $R D$ of $R$ on $P$ is not null.

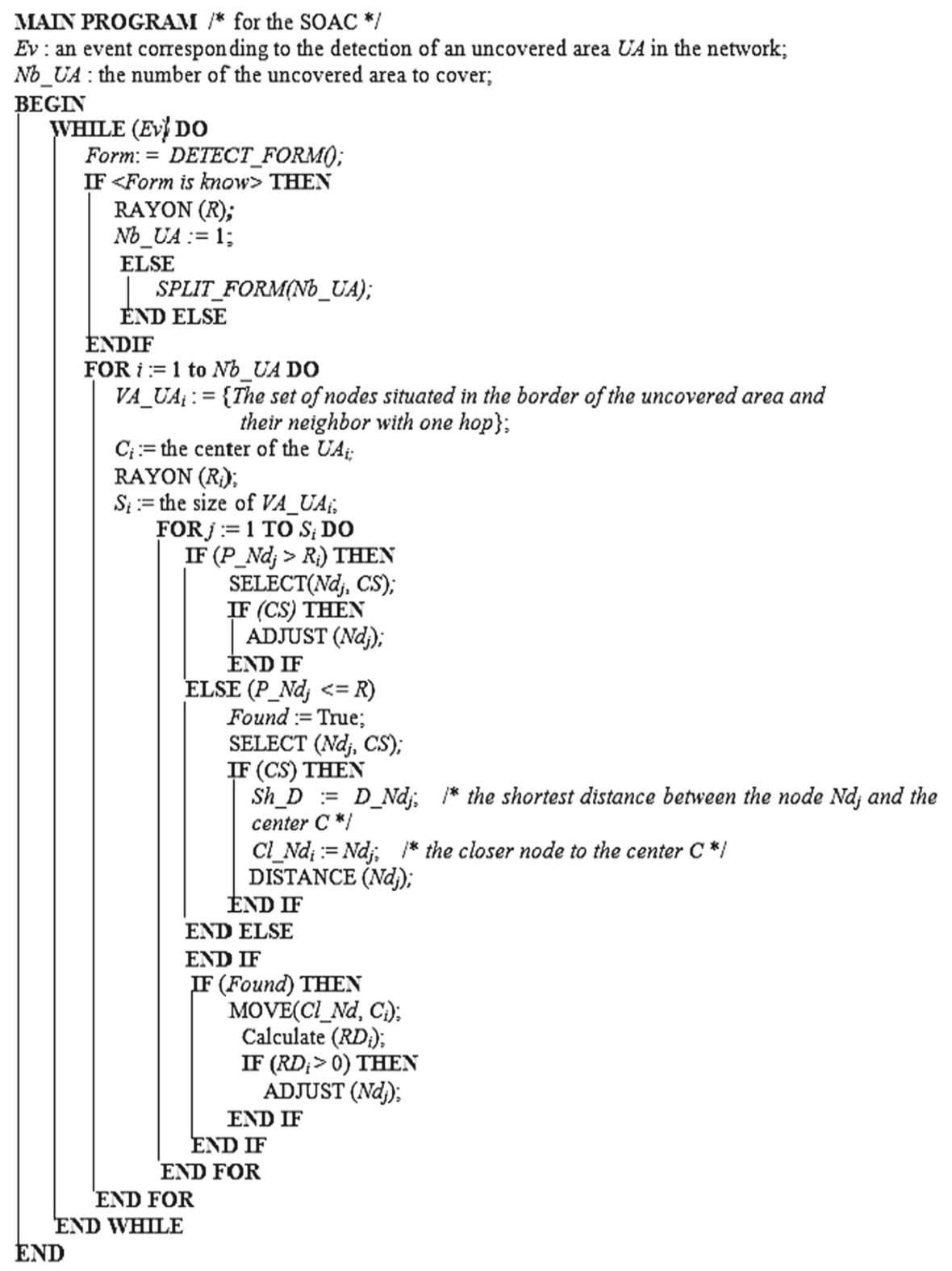

Algorithm 1. The main module of SOAC technique 
Figure 1 gives graphical version of the Algorithm 1 with the main steps of the functioning of our SOAC proposed technique.

The module (or agent) containing our proposed SOAC solution is triggered by receiving an event signaling a possible appearance of uncovered zone within the network. Such event can be generated by any node from the network but in our case we have only considered source or destination nodes of the data packets. When a source node fails to join some nodes all located in the same place of a network, or when a destination node receives information expected from a node located in the same zone, it is more likely that this zone will be unreachable or uncovered. According to the information obtained by SOAC about the unreachable nodes, mainly their last geographical coordinates, SOAC determines the geometric form of the uncovered zone that has just appeared in the network. Two cases are distinguished, either the geographic form is known (like triangle, circle, square, etc.) or it is not known. In the case where the form of the gap (area) is known, then the radius of this area is calculated by the procedure $R A Y O N()$ and the number of uncovered area is set to 1 $\left(N b_{-} U A:=1\right)$. Otherwise, the uncovered zone will then be divided into several zones with known forms and this with the procedure $S L I P T\left(\right.$ ) (with $N b_{-} U A<>1$ ). In both cases, the same treatment will be applied to each uncovered area.

After calculating the radius $R$ of the uncovered area, it will be then compared to the communication range $P$ set by default. Two cases are also distinguished, $P$ is greater than $R$ or $P$ is less than $R$. In the first case $(P>R)$, SOAC search all the nodes situated at the border of the uncovered zone that satisfy the condition $C S$ given by Eq. (1). Among all of these nodes, SOAC select the closest one to the uncovered area center $(C)$ and moves it to $C$ position. After, the difference between $P$ and $R$ is calculated according to the Eq. (3) and if obtained $R D$ is not null, the communication range $P$ of the moved node is also adjusted according to Eq. (2). In the second case $(P<R)$, SOAC selects first all the nodes situated at the border of the uncovered zone that satisfy the condition CS given by Eq. (1). After that, the communication range $P$ of all these nodes is adjusted according to the Eq. (2). All the treatments that we have just explained are repeated for all the uncovered areas detected. SOAC provides an efficient and intelligent solution to reorganize the network and improves the network coverage quality.

\section{Performance evaluation}

In this section we introduce firstly the simulation environment and then we present the obtained results when using our proposed technique. We are interested firstly in the quality of network coverage achieved by SOAC. Secondary, SOAC is evaluated in term of some QoS parameters mainly the end to
Table 1 The default parameters used in the simulations

\begin{tabular}{ll}
\hline Parameters & Values \\
\hline Position of sensors & Random \\
Size of the network & $1200 \mathrm{~m} \times 1200 \mathrm{~m}$ \\
Simulation time & $100 \mathrm{mn}$ \\
MAC layer & IEEE $802.11 \mathrm{a}$ \\
Physical layer & OFDM \\
Transport layer & TCP NewReno \\
Network layer & AODV \\
Inter packet interval & $2 \mathrm{~s}$ \\
Propagation model & Two-ray ground model \\
Packets scheduling & FIFO \\
Bandwidth & $1 \mathrm{Mb} / \mathrm{s}$ \\
Queue buffer & 50 packets \\
Transmission range & $50 \mathrm{~m}$ \\
Interference range & $100 \mathrm{~m}$ \\
Initial energy/node & $1000 \mathrm{~J}$ \\
Packet size & 512 bytes
\end{tabular}

end delay, the evolution of the consumed energy and the lifetime of the nodes. The obtained results for the network using SOAC are compared to those of the network without any solution but also to those recorded by the networks using the solutions proposed in [20] (solution1) and in [22] (solution2).

\subsection{Simulation environment}

The simulations are performed under OMNET++ simulator [23] and each one is repeated several times in order to obtain a 95\% confidence interval (for 100 repeated simulations, 95 of them give the same results). For the initial positions of the nodes, we used BonnMotion tool [24] allowing uniform distribution and the generation of the topology. Based on the works done in [25-27], we decided to exploit some existing communication protocols for the implementation of SOAC. In fact, the results of these works showed good performance in the context of wireless networks. The distributed coordination function (DCF) of the 802.11a medium access control (MAC) protocol is used with the default parameters used by this function. As transmission control protocol (TCP), we decided to use New Reno version and due to the ad hoc nature of the considered networks, we decided to use ad hoc on demand vector distance (AODV) as routing protocol. The mobility model uses the random waypoint with a speed of $5 \mathrm{~m} / \mathrm{s}$ and time pause of $10 \mathrm{~s}$. A simple energy model is used decreasing the level of the node by one unit for every sent packet $(0.001 \mathrm{~J} /$ packet $)$.

Table 1 gives the default parameters of the environment of the simulations the most important ones considered for the evaluation of our SOAC proposed technique. 

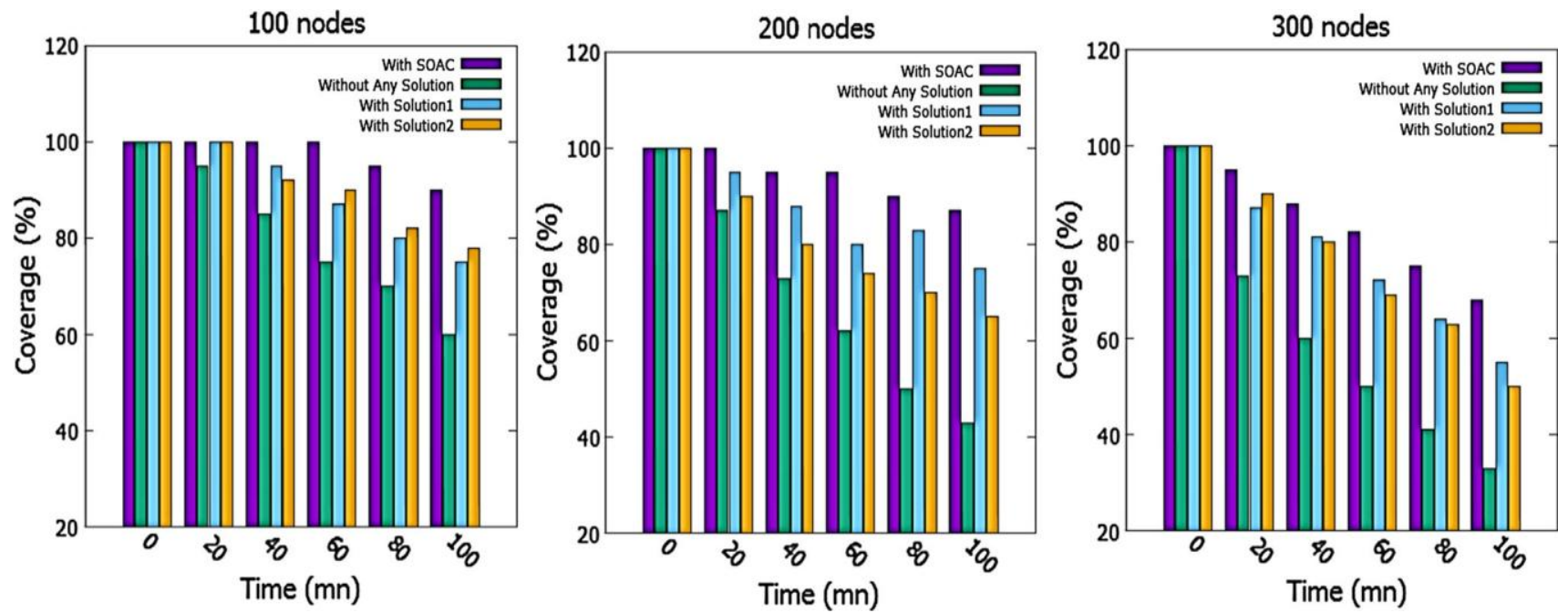

Fig. 2 The variation of network coverage with time for 100, 200 and 300 nodes

\subsection{Network coverage performance}

In terms of network coverage, we see through Fig. 2 that the number of nodes used in the network influences the total network coverage. We also note that the network using the proposed SOAC technique performs better compared to the other three networks. These results remain the same for the three scenarios studied (100, 200 and 300 nodes). In the first scenario with 100 nodes, the network using SOAC maintains $100 \%$ of the coverage up to minute 80 of the simulation. Contrary to the other networks with which the network coverage begins to decrease much earlier.

For example the coverage of the network without any solution has decreased by $5 \%$ at minute 20 of the simulation and by $30 \%$ at minute 80 . Even the solution 1 and the solution 2 recorded better results compared to network without solution; these results are less efficient than those obtained with SOAC. At the end of the simulation, SOAC is the best in terms of network with $90 \%$ of network coverage. Solution2 comes the second with $78 \%$ of coverage and after solution 1 with $75 \%$. The network without any solution recorded at the end of the simulation $60 \%$ of network coverage.

The proposed solution is the best in terms of network coverage even for scenarios with 200 and 300 nodes. With 200 nodes and at the end of the simulation, SOAC improves the network coverage by $44 \%$ compared to the network without any solution, by $12 \%$ compared to the solution 1 and by $22 \%$ compared to solution2. Same for the scenario with 300 nodes where our solution recorded at the end of the simulation, an improvement of $13 \%$ compared to solution 1 and $18 \%$ compared to solution2.

In fact, the degradation of network coverage, especially for the networks that does not use SOAC technique, is mainly due to the activity stop of the nodes after the exhaustion of their energy. This leads to the formation of uncovered areas which are degrading the total network coverage. The good results obtained with our proposed technique, even with the same environment, is due to the fact that it is supporting a mechanism allowing a self-organization of the network in order to cover the detected uncovered area. In fact, this selforganization is realized by acting on the parameters of some nodes with ensuring their fair exploitation and evolution. Based on these results we can say that SOAC improves the performance of the CMONs in terms of network coverage.

\subsection{End to end delay performance}

Figure 3 shows the evolution of the end to end delay for the sent data with three different scenarios. We can see clearly through the graphs that the more the number of nodes increases in the network the more the end to end delay recorded increases. However we see a difference in performance between the different networks and when the SOAC technique is used the obtained results are better. For example, for the scenario with 100 nodes, $95.3 \%$ of all the sent data with the network using SOAC recorded an end to end delay inferior to $50 \mathrm{~ms}$. Only $0.2 \%$ of the sent data with the same network recorded an end to end delay which exceeds $150 \mathrm{~ms}$. However, the networks using solution1 and solution2 recorded higher end to end delay values compared to those obtained with the network using SOAC. Only $77.0 \%$ of the sent data with the network using solution1 recorded an end to end delay inferior to $50 \mathrm{~ms}$. For the network using solution2, $78.5 \%$ of sent data recorded an end to end delay inferior to $50 \mathrm{~ms}$.

Even with the two other scenarios, using 200 and 300 nodes, the proposed technique stays the best one in terms of end to end delay performance. The end to end delay 

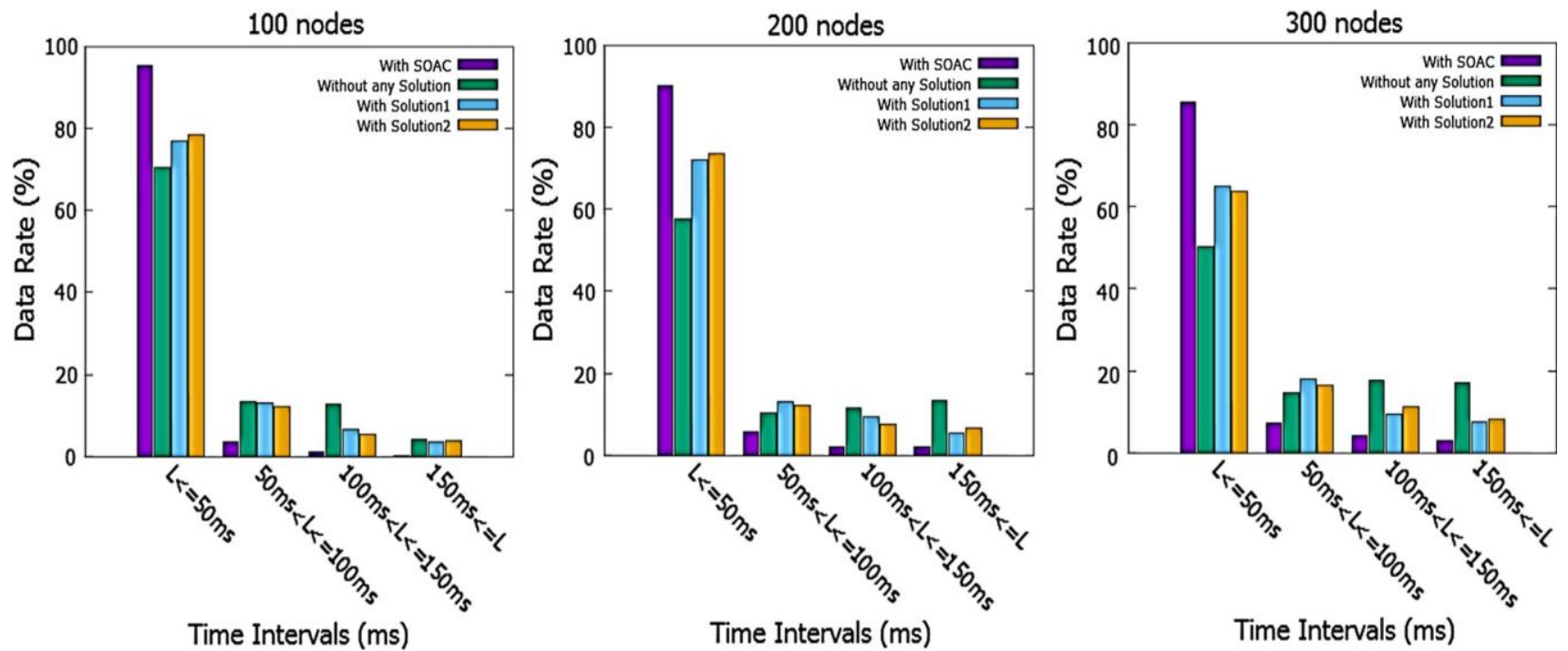

Fig. 3 The variation of end to end delay with time for 100, 200 and 300 nodes
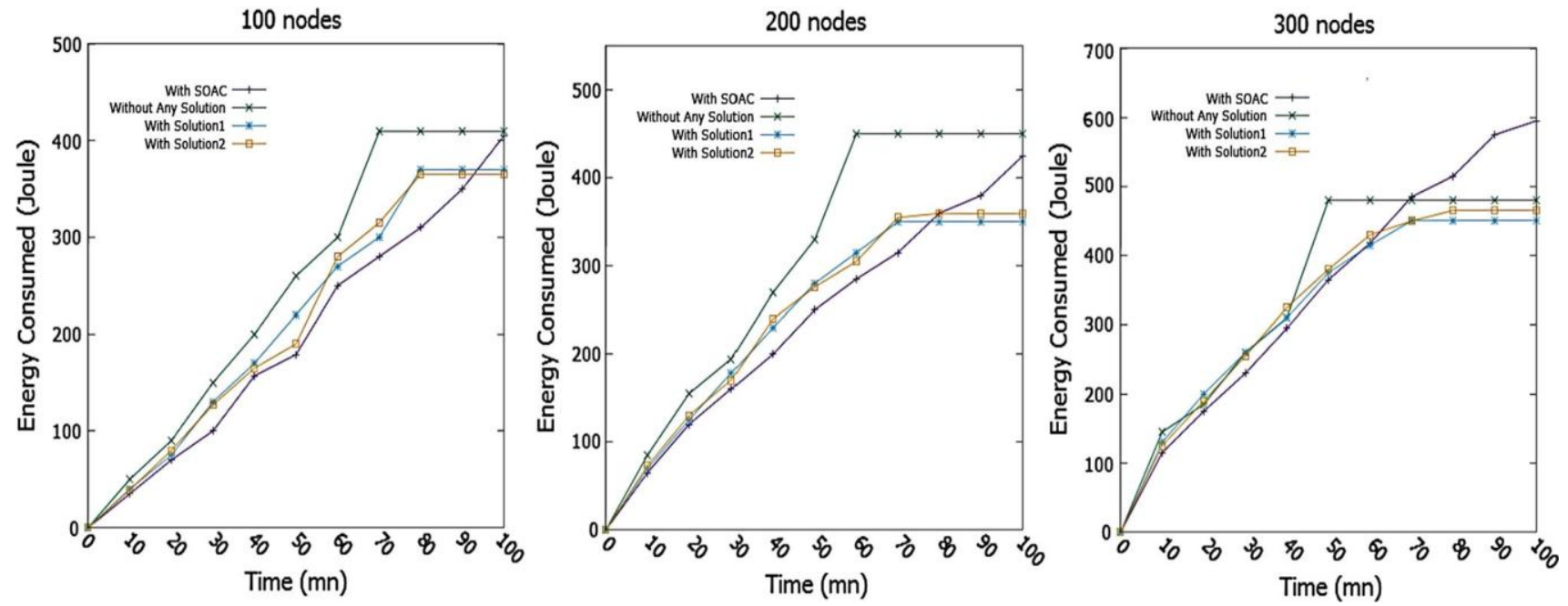

Fig. 4 The variation of the consumed energy with time for 100, 200 and 300 nodes

of $90.1 \%$ of all the sent data is inferior to $50 \mathrm{~ms}$ (with $1 \mathrm{~ms}=0.000016 \mathrm{mn}$ ) with the network using SOAC and containing 200 nodes. With the same scenario, only $2 \%$ of the sent data recorded an end to end delay exceeding $150 \mathrm{~ms}$. These results remain better compared to those obtained with the network without any solution and with those using solution1 and solution3. Our technique keeps its performance also with the last scenario (with 300 nodes). The performance improvement recorded with the network using SOAC is due to the maximization of the network coverage ensured. By reducing the uncovered area appeared in the network, SOAC reduces then data losses and avoid additional delay for the retransmission of these data. An important factor which leads to improve the end to end delay considered as basic QoS parameters for the CMONs applications.

\subsection{Consumed energy performance}

Figure 4 shows the evolution of consumed energy over time for four different networks. We can see for all the networks that the amount of the energy used increases with time. The network without any solution consumes more energy compared to the networks using solution 1 and solution 2 which consume more energy than the network using SOAC technique. We can also see that the increase in consumed energy is slowly with SOAC in comparison to the others networks. Through the obtained results we can also say that the number of nodes has an impact on the amount of the consumed energy. The network containing 100 nodes, and without any solution, sees its consumed energy stabilize at almost $400 \mathrm{~J}$ and this at minute 70 of the simulation. The same observation for the networks using solution 1 and solution 2 with which the 

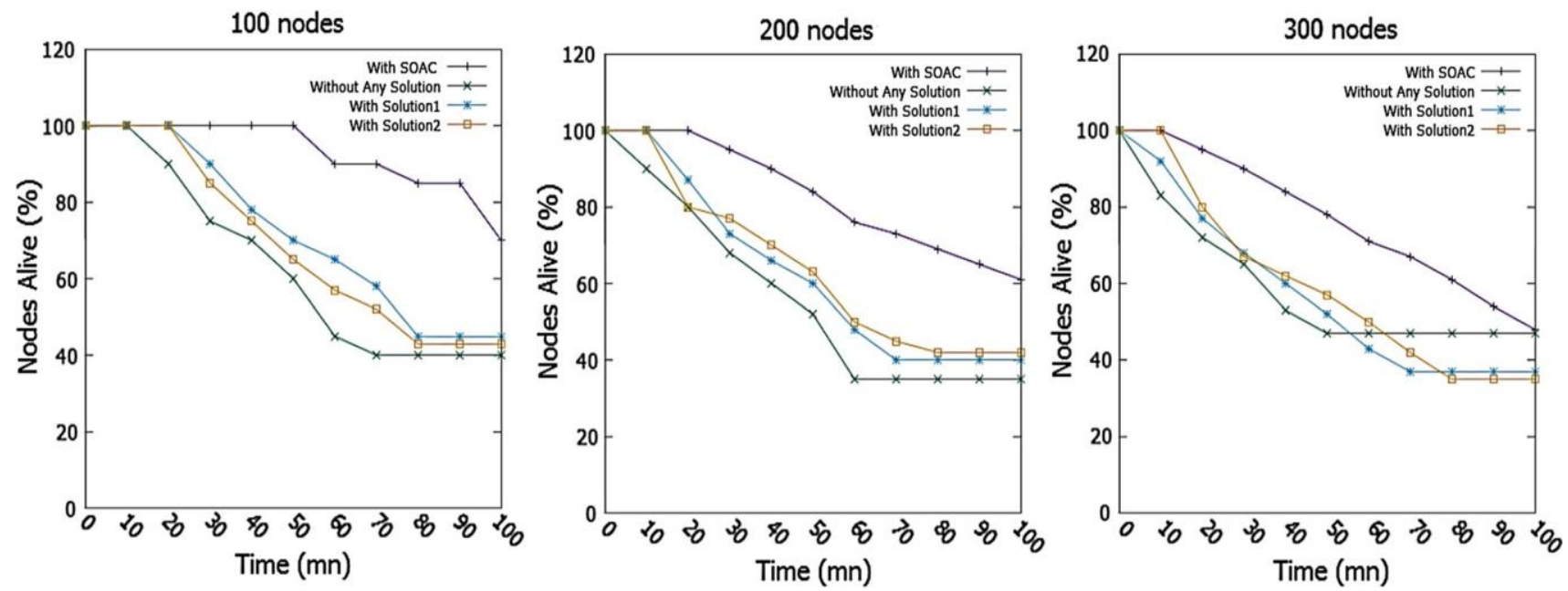

Fig. 5 The variation of lifetime of nodes with time for 100, 200 and 300 nodes

consumed energy stops increasing and stabilizes with almost $350 \mathrm{~J}$ and this at minute 80 of the simulation. However, the network using our solution continues to consume normally the energy until the last minute of the simulation.

With the two others scenarios using more number of nodes, SOAC remains the best in recording communication activity until the end of the simulation. Contrary to the other networks with which the consumed energy stops increasing earlier than the previous scenario (with 100 nodes). In fact, the stabilization of the energy with the three first networks is due to the stop of the communication activity. The apparition of uncovered areas in the networks leads to the connectivity break between nodes and then the losing of data and the impossibility of keeping communication between them. This problem is reduced by the mechanism of self-organization implemented by SOAC allowing the maximization of the network coverage and the fair evolution of consumed energy between nodes. Such features keep up the network activity until the end of simulation and allow a fair evolution of the energy.

\subsection{Nodes lifetime performance}

Figure 5 gives the evolution of the percentage of active nodes over time. We can see that the percentage of active nodes in the network without any solution and the networks using solution1 and solution2 begins to decrease earlier than in the network using SOAC. For the scenario with 100 nodes, the percentage of active nodes in the network without any solution stabilizes at $40 \%$ between minute 70 and the last minute of simulation. This stabilization is the result of the activity (communication) cessation in the network and this after detecting the connectivity breaks between some nodes and the appearance of many uncovered areas. The same observation for the networks using the solution1 and solution 2 where the percentage of active nodes stabilizes at $45 \%$ and at $43 \%$ respectively and this from minute 80 of the simulation. However, we see a continued decrease in the percentage of active nodes in the network using SOAC until the last minute of the simulation where $70 \%$ of the nodes are still alive.

For the two other scenarios, with 200 and 300 nodes, the network using SOAC keeps its best performance in terms of percentage of nodes alive compared to the three other networks. In both scenarios, the percentage of the nodes which are alive begins to decline earlier than in the previous scenario (with 100 nodes). The percentage of nodes which are still alive stabilizes earlier compared to the previous scenario. The network using SOAC gets to keep alive nodes even until the last minute of the simulation with $60 \%$ of nodes that are still alive for the second scenario and $48 \%$ for the last scenario. In fact, with the increase in the number of nodes in the network (from 100 to 300), there is an increase in the interferences and in packet losses generated by the nodes. Due to its ability to take into account the communication parameters, which can be sources of data losses, these interferences are less important with SOAC. This is why we have obtained better results with our technique compared to the other networks.

Through these last results, we conclude that SOAC offers a solution to the problem of connectivity breaks caused by the disappearance of some nodes in the network allowing then to minimize the uncovered areas. The network using SOAC recorded always better results compared to the other networks. Such result is already a strong factor which contributes in the improvement, in addition to coverage quality, important QoS parameters for CMONs applications. 


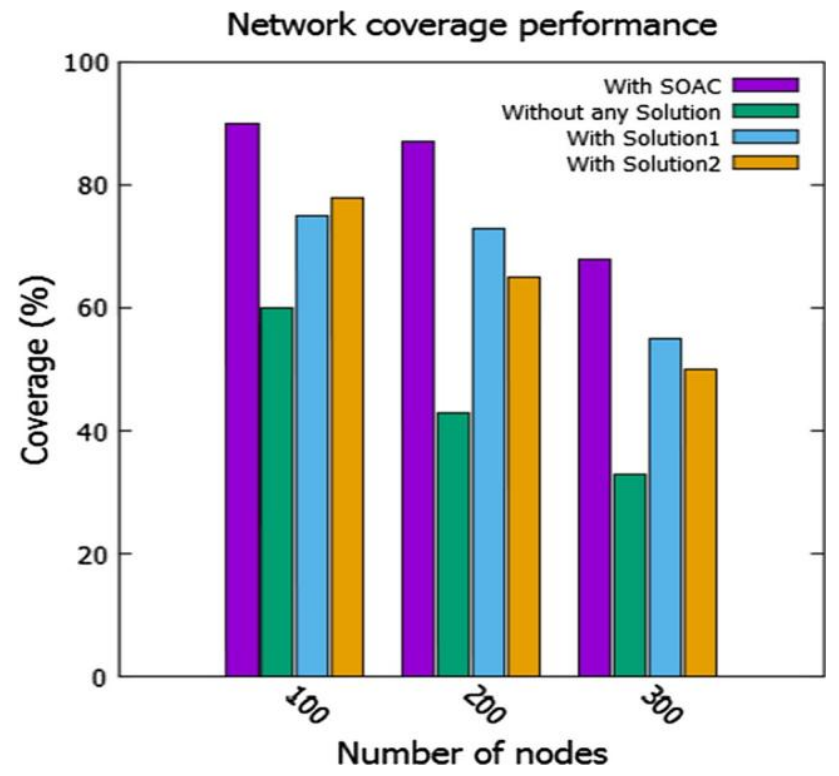

Fig. 6 The variation of network coverage according to the number of nodes

\subsection{Evolution of the SOAC performance with the number of nodes}

We are proposing in this section to evaluate our proposed solution according to the number of nodes used by the network. In each one of the previous simulations, we give the graphs of the obtained results by all the solutions at the end of the simulation. For the end to end delay, we represent only the sent packets rate at the interval $[0,50 \mathrm{~ms}]$. The evolution of network coverage rate is given by Fig. 6 . The more the number of nodes is important the more will be the degradation of network coverage. This degradation is associated usually to the important occurred interferences in the network leading to resend more often the data. Some nodes will stop working after completely consuming their energy. This constitutes the main reason for the appearance of uncovered areas in the network reducing then total network coverage, as showed in Fig. 6. However, the degradation of coverage recorded with SOAC solution is minor compared to the three other networks. Even with 300 nodes, SOAC has allowed to cover $68 \%$ of the network.

Figure 7 gives the evolution of the end to end delay for SOAC varying with the number of nodes compared to the three other solutions considered in our paper. We can notice that the end to end delay is influenced by the number of nodes in the network. This influence is less important with SOAC technique which has recorded some $85.4 \%$ end to end delay even when the network uses 300 nodes.

The consumed energy and lifetime of nodes are two related parameters that are also influenced by a large number of nodes. The increase in the consumed energy and the reduction

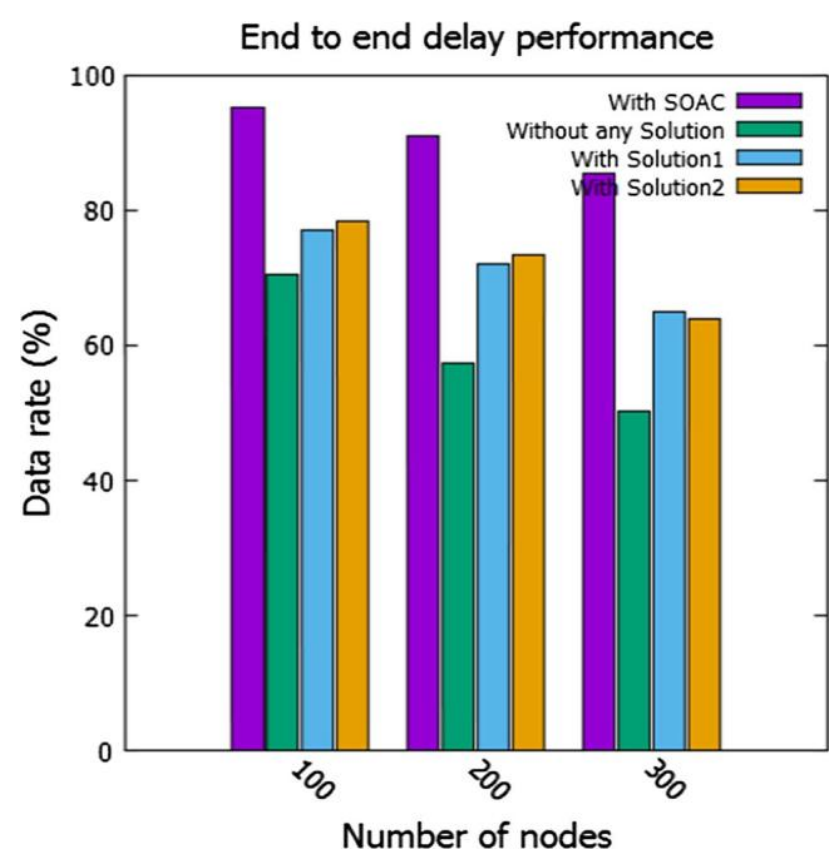

Fig. 7 The variation of end to end delay according to the number of nodes

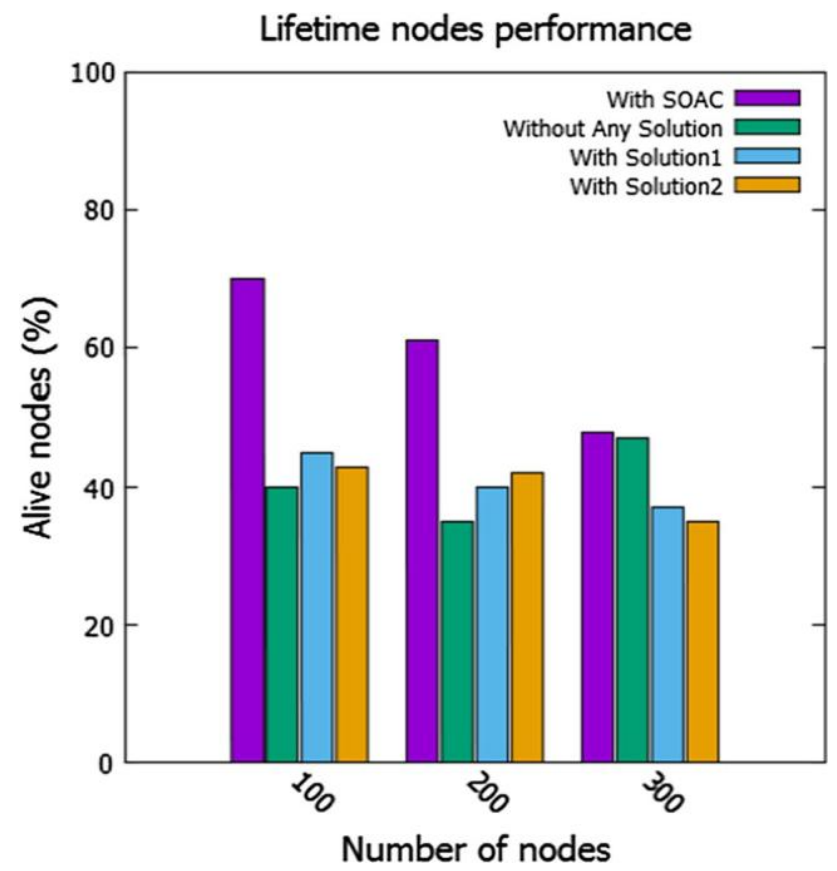

Fig. 8 The variation of lifetime of nodes according to the number of nodes

of active nodes are two factors for the presence of activity in the network. But when these two parameters stop changing this generally is due to some activity stop in the network. This is often caused by the appearance of uncovered areas in the network. Even with the presence of some actives nodes in the network, they are not able to be connected and communicate. 


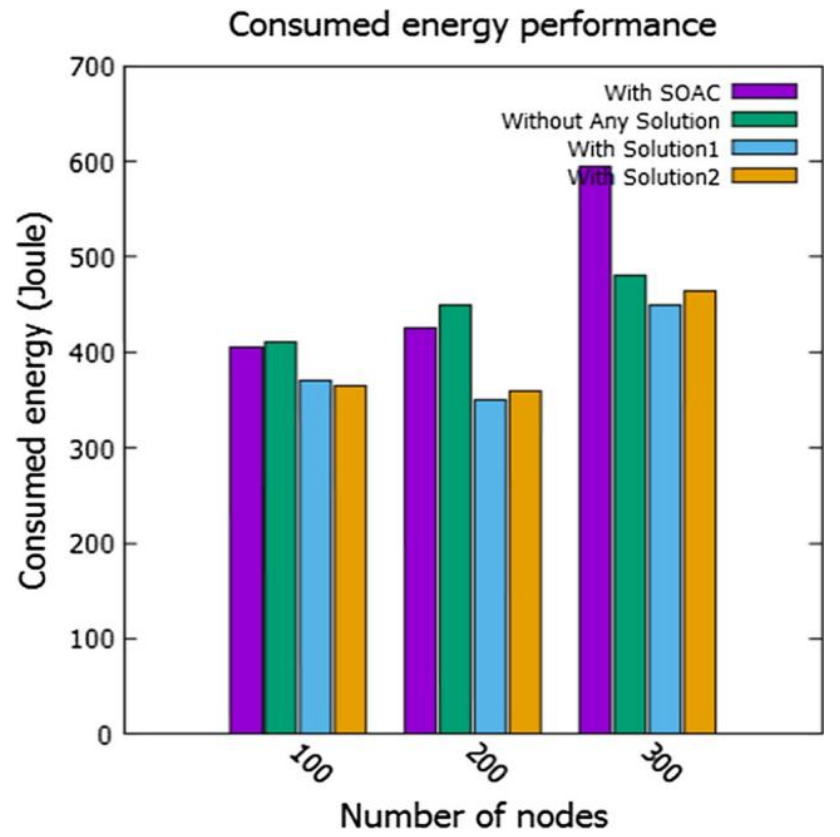

Fig. 9 The variation of consumed energy according to the number of nodes

Figures 8 and 9 illustrate the performance results obtained with our proposed technique when compared to the three other solutions studied. Despite a large number of nodes in the network, SOAC maintains its performance in terms of energy consumption and nodes lifetime. Unlike other solutions, and even 300 nodes, SOAC gets to keep $48 \%$ of the active nodes.

Same for the consumed energy, the network using SOAC recorded slightly larger quantities than the three others networks. This is due to the fact that SOAC maintains the activity in the network until the last minute of simulation even with a large number of nodes. For example, with 300 nodes, SOAC recorded almost $600 \mathrm{~J}$ at the last minute of simulation.

\section{Conclusion and perspectives}

The work presented in this paper is situated at the intersection of two important areas namely the CMONs and self-organizing systems. We started by presenting the proposed SOAC technique which aims to improve the global coverage within CMONs while optimizing the exploited resources. We act directly on the connected nodes by changing two of their parameters which are the mobility and the communication range. We used simulations in order to evaluate the performance of SOAC and performed several scenarios with different number of nodes. Important metrics have been considered namely network coverage, end-to-end delay, lifetime of the nodes and energy consumption. For the different simulations and with the all scenarios studied,
SOAC has recorded an important improvement compared to three others solutions proposed in the same context.

Our future work consists in the extension of proposed SOAC technique with the integration of more communication parameters in order to reflect as much as possible real situations which can be present in CMONs. This work is also open to add some geometrics and mathematical techniques to better take into account the case when the form of uncovered area appearing in the network is complex. In the end, our proposed technique can be exploited in other areas such as intelligent houses and cities, e-health, intelligent transport and vehicles communications.

\section{References}

1. Lloret, J., Tomás, J., Garcia, M., \& Cánovas, A. (2009). A hybrid stochastic approach for self-location of wireless sensors in indoor environments. Sensors, 9(5), 3695-3712.

2. Garcia, M., Martinez, C., Tomas, J., \& Lloret, J. (2007). Wireless sensors self-location in an indoor WLAN environment. In International conference on sensor technologies and applications, SENSORCOMM 2007, October 14-20, 2007, Valencia, Spain.

3. Garcia, M., Tomas, J., Boronat, F., \& Lloret, J. (2009). The development of two systems for indoor wireless sensors self-location. Ad Hoc \& Sensor Wireless Networks, 8(3-4), 235-258.

4. Sendra, S., García, M., Turró, C., \& Lloret, J. (2011). WLAN IEEE $802.11 \mathrm{a} / \mathrm{b} / \mathrm{g} / \mathrm{n}$ indoor coverage and interference performance study. International Journal on Advances in Networks and Services, 4(1), 209-222.

5. Lara, R., Benitez, D., Zennaro, A. C., \& Rojo-Alvarez, J. (2015). On real-time performance evaluation of volcano monitoring systems with wireless sensor networks. Sensors Journal, 15(6), 3514-3523.

6. Sendra, S., Fernandez, P., Turro, C., \& Lloret, J. (2010). IEEE $802.11 \mathrm{a} / \mathrm{b} / \mathrm{g} / \mathrm{n}$ indoor coverage and performance comparison. In The sixth international conference on wireless and mobile communications. ICWMC 2010. September 20-25, 2010-Valencia, Spain.

7. Abdul-Salaam, G., Abdullah, A. H., Anisi, M. H., Gani, A., \& Alelaiwi, A. (2016). A comparative analysis of energy conservation approaches in hybrid wireless sensor networks data collection protocols. Telecommunication Systems, 61(1), 159-179.

8. Natalizio, E., \& Loscri, V. (2013). Controlled mobility in mobile sensor networks: Advantages, issues and challenges. Telecommunication Systems, 52(4), 2411-2418.

9. Liu, J., Chen, Y., Chen, X., Ding, J., Chowdhury, K. R., Hu, Q., et al. (2013). A cooperative evolution for QoS-driven IoT service composition. Automatika, 54(4), 438-447.

10. Akkaya, K., Senel, F., Thimmapuram, A., \& Uludag, S. (2010). Distributed recovery from network partitioning in movable sensor/actor networks via controlled mobility. IEEE Transactions on Computers, 59(2), 258-271.

11. Anzola, J. P., Bolaños, S. J., \& Bermúdez, G. M. T. (2016). Design methodology for self-organized mobile networks based. International Journal of Interactive Multimedia and Artificial Intelligence, $3(7), 46-53$.

12. Saha, D., \& Das, N. (2016). Self-organized area coverage in wireless sensor networks by limited node mobility. Innovations in Systems and Software Engineering, 12, 227-238.

13. Hamrioui, S., \& Lorenz, P. (2015). ES-WSN: Energy efficient by switching between roles of nodes in WSNs. In Proceedings of IEEE 
GloCom'15, SAC: Internet of Things, Dec. 6-10, 2015, San Diego, CA, USA.

14. Hamrioui, S., \& Lorenz, P. (2016). EQ-AODV: Energy and QoS supported AODV for better performance in WMSNs. In Proceedings of IEEE ICC 2016, SAC:E-Health, 23-27 May 2016, Kuala Lumpur, Malaysia.

15. Yang, T., Kang, C. J., \& Nan, G. (2012). An energy-efficient and fault-tolerant convergecast protocol in wireless sensor networks. International Journal of Distributed Sensor Networks, 8, 1-8.

16. Zou, Y., \& Chakrabarty, K. (2007). Redundancy analysis and a distributed self-organization protocol for fault-tolerant wireless sensor networks. International Journal of Distributed Sensor Networks, 3(3), 243-272.

17. Gajjar, S., Sarkar, M., \& Dasgupta, K. (2014). Self-organized, flexible, latency and energy efficient protocol for wireless sensor networks. International Journal of Wireless Information Networks, 21(4), 290-305.

18. Bajo, J., Paz, J. F. D., Villarrubia, G., \& Corchado, J. M. (2015). Self-organizing architecture for information fusion in distributed sensor networks. International Journal of Distributed Sensor Networks, 11, 1-13.

19. Deepa, O., \& Visalakshi, N. K. (2016). A self-organized QoSaware RED-ACO routing protocol for wireless sensor networks. Middle-East Journal of Scientific Research, 24, 224-230.

20. Gao, Y., Wkram, C. H., Duan, J., \& Chou, J. (2015). A Novel energy-aware distributed clustering algorithm for heterogeneous wireless sensor networks in the mobile environment. Sensors, 15, 31108-31124.

21. Costanzo, C., Loscri, V., Natalizio, E., \& Razafindralambo, T. (2012). Nodes self-deployment for coverage maximization in mobile robot networks using an evolving neural network. Computer Communications, 35(9), 1047-1055.

22. Abbasi, M., AbdLatiff, M. S., Modirkhazeni, A., \& Anisi, M. H. (2011). Optimization of wireless sensor network coverage based on evolutionary algorithm. International Journal Computer Communications and Networks, 1, 1-5.

23. Varga, A. (2001). The OMNeT++ discrete event simulation system. In Proceedings of the European simulation multiconference (ESM 2001), Prague, Czech Republic, 6-9 June 2001.

24. Aschenbruck, N., Ernst, R., Padilla, E.G., \& Schwamborn, M. (2010). BonnMotion: A mobility scenario generation and analysis tool. In Proceedings of the 3rd international ICST conference on simulation tools and techniques, Malaga, Spain, 15-19 June 2010.

25. Hamrioui, S., Lorenz, P., LIoret, J., \& Lalam, M. (2014). Energy efficient in medical ad hoc sensors network by exploiting routing protocols. In IEEE Globecom 2014: Symposium on selected areas in communications-GC14 SAC e-Health, TX, USA (pp. 24172422).

26. Hamrioui, S., Lorenz, P., Lloret, J., Rodrigues, J. J. P. C., \& Lalam, M. (2015). Chapter 2: Network planning and designing. In M. Obaidat, P. Nicopolitidis, \& F. Zarai (Eds.). Modeling and simulation of computer networks and systems methodologies and applications (pp. 33-53). Amsterdam: Elsevier. ISBN: 978-0-12-800887-4.

27. Hamrioui, S., Lorenz, P., \& Rodrigues, J. J. P. C. (2014). Mobile ad hoc networks performance: Interactions between routing and transport protocols. Saarbrücken: LAP LAMBERT Academic Publishing, OmniScriptum GmbH \& Co. KG. ISBN: 978-3-65962608-1.

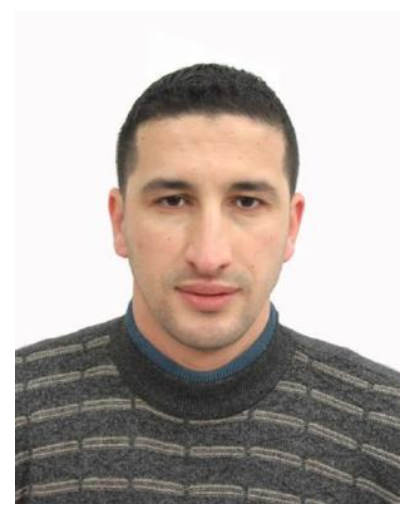

Sofiane Hamrioui received his Engineer degree (2004), Magister (2007) and Ph.D. (2014) from the University of Mouloud Mammeri, Tizi Ouzou, Algeria. Between 2008 and 2014 he was a researcher and teacher at University Mouloud Mammeri of Tizi Ouzou and at the University of Sciences and Technologies Houari Boumediene, Algiers, Algeria. He was an assistant professor at the University of Sciences and Technologies Houari Boumediene, Algiers, Algeria, between 2014 and 2015. Since the end of 2015, he is teacher and researcher at the University of Haute Alsace, France. His main research has been focused on designing and developing network protocols and algorithms, especially on QoS-based wireless networks. These designs have been used in many real environments using P2P networks, Ad-hoc and sensor networks, Grids and Content Distribution Networks. Until 2016, he had more than (20) scientific papers published in national and international conferences, he had more than (10) papers published in international journals and he was the author/co-author of 4 books. He has been a guest editor for IEEE Transactions on Emerging Topics in Computing and he is editorial board member of the Network Protocols and Algorithms journal. He was a chair of (02) international workshops and (01) special session in an international conference. He has served as Technical committee member for more than (20) international conferences.

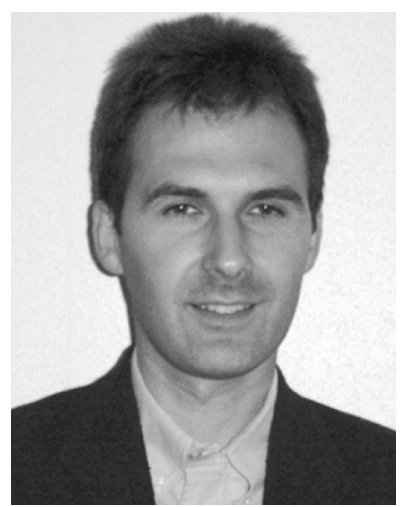

Pascal Lorenz received his M.Sc. (1990) and Ph.D. (1994) from the University of Nancy, France. Between 1990 and 1995 he was a research engineer at WorldFIP Europe and at AlcatelAlsthom. He is a professor at the University of Haute-Alsace, France, since 1995. His research interests include QoS, wireless networks and high-speed networks. He is the author/co-author of 3 books, 3 patents and 200 international publications in refereed journals and conferences.

He was Technical Editor of the IEEE Communications Magazine Editorial Board (2000-2006), Chair of Vertical Issues in Communication Systems Technical Committee Cluster (2008-2009), Chair of the Communications Systems Integration and Modeling Technical Committee (2003-2009) and Chair of the Communications Software Technical Committee (2008-2010). He has served as Co-Program Chair of IEEE WCNC'2012, ICC'2004 and ICC'2017, tutorial chair of VTC'2013 Spring and WCNC'2010, track chair of PIMRC'2012, symposium Co-Chair at Globecom 2007-2011, ICC 2008-2010, ICC'2014 and '2016. He has served as Co-Guest Editor for special issues of IEEE Communications Magazine, Networks Magazine, Wireless Communications Magazine, Telecommunications Systems and LNCS. He is associate Editor for International Journal of Communication Sys- 
tems (IJCS-Wiley), Journal on Security and Communication Networks (SCN-Wiley) and International Journal of Business Data Communications and Networking, Journal of Network and Computer Applications (JNCA-Elsevier). He is senior member of the IEEE, IARIA fellow and member of many international program committees. He has organized many conferences, chaired several technical sessions and gave tutorials at major international conferences. He was IEEE ComSoc Distinguished Lecturer Tour during 2013-2014.

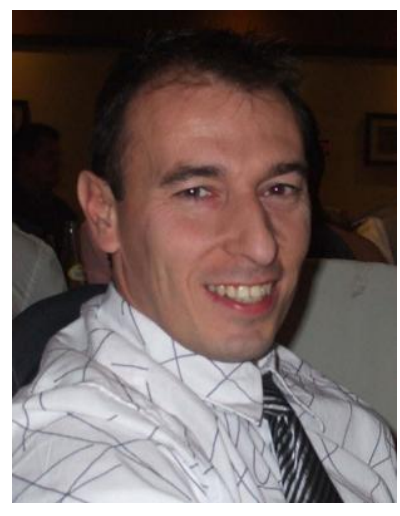

Jaime Lloret received his M.Sc. in Physics in 1997 at University of Valencia and he finished a postgraduate Master in Corporative networks and Systems Integration from the Department of Communications in 1999. Later, he received his M.Sc. in Electronic Engineering in 2003 at University of Valencia and his $\mathrm{Ph} . \mathrm{D}$. in telecommunication engineering (Dr. Ing.) at the Polytechnic University of Valencia in 2006. Before concluding his Ph.D. Thesis he obtained the first place given by the Spanish Agency for Quality Assessment and Accreditation for the Campus of Excellence in the New Technologies and Applied Sciences Area. He was awarded the prize of the best doctoral Student in the Telecommunications area in 2006 according to the Social Council of the Polytechnic University of Valencia. He is a Cisco Certified Network Professional Instructor of the regional academy "Universidad Politécnica de Valencia" in the Cisco Networking Academy Program (CNAP) and he is the Legal Main Contact of UPV-ADIF (local academy of the CNAP). He teaches Local Area Networks and Systems Integration in the "Escuela Politecnica Superior de Gandia" from the Polytechnic University of Valencia. He has been working as a network designer and administrator in several companies. His academic interests and research are P2P networks, Wireless Local Area Networks, Sensor Networks and Routing Protocols. He also researches on educational approaches and strategies. 